\title{
Monotone iterative technique for nonlinear differential equation of fractional order
}

\author{
Zhengzhi $\mathrm{Lu}^{1}$, Li Yongjun ${ }^{1}$, and Xiaoyan $\mathrm{Shi}^{2}$ \\ ${ }^{1}$ Lanzhou City University \\ ${ }^{2}$ Southwestern University of Finance and Economics
}

May 26, 2021

\begin{abstract}
In this paper, we mainly study the existence of solution of fractional differential equations. Firstly, the existence of the maxmum solution and minmum solution of the differential equation are proved by using the fixed point theorem and the monotone iteration method. Secondly, the existence of the solution of the original equation is proved by using the newly constructed differential equation. Finally, the application of the monotone iteration method is given through an example.
\end{abstract}

\section{Hosted file}

Monotone iterative -MMAS.pdf available at https://authorea.com/users/369687/articles/523605monotone-iterative-technique-for-nonlinear-differential-equation-of-fractional-order 